Corrigendum

\title{
Corrigendum to "Hydrogen Sulfide Prevents Formation of Reactive Oxygen Species through PI3K/Akt Signaling and Limits Ventilator-Induced Lung Injury"
}

\author{
Sashko Georgiev Spassov, Rosa Donus, Paul Mikael Ihle, Helen Engelstaedter, \\ Alexander Hoetzel, and Simone Faller
}

Department of Anesthesiology and Critical Care, Medical Center-University of Freiburg, Faculty of Medicine, University of Freiburg, Freiburg, Germany

Correspondence should be addressed to Simone Faller; simone.faller@uniklinik-freiburg.de

Received 6 August 2017; Accepted 20 September 2017; Published 16 October 2017

Copyright (c) 2017 Sashko Georgiev Spassov et al. This is an open access article distributed under the Creative Commons Attribution License, which permits unrestricted use, distribution, and reproduction in any medium, provided the original work is properly cited.

In the article titled "Hydrogen Sulfide Prevents Formation of Reactive Oxygen Species through PI3K/Akt Signaling and Limits Ventilator-Induced Lung Injury" [1], there was an error in the authors' affiliation. The corrected affiliation is shown above.

\section{References}

[1] S. G. Spassov, R. Donus, P. M. Ihle, H. Engelstaedter, A. Hoetzel, and S. Faller, "Hydrogen sulfide prevents formation of reactive oxygen species through PI3K/Akt signaling and limits ventilator-induced lung injury," Oxidative Medicine and Cellular Longevity, vol. 2017, Article ID 3715037, 14 pages, 2017. 


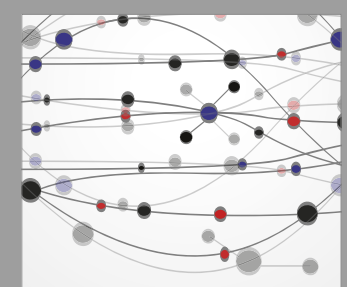

The Scientific World Journal
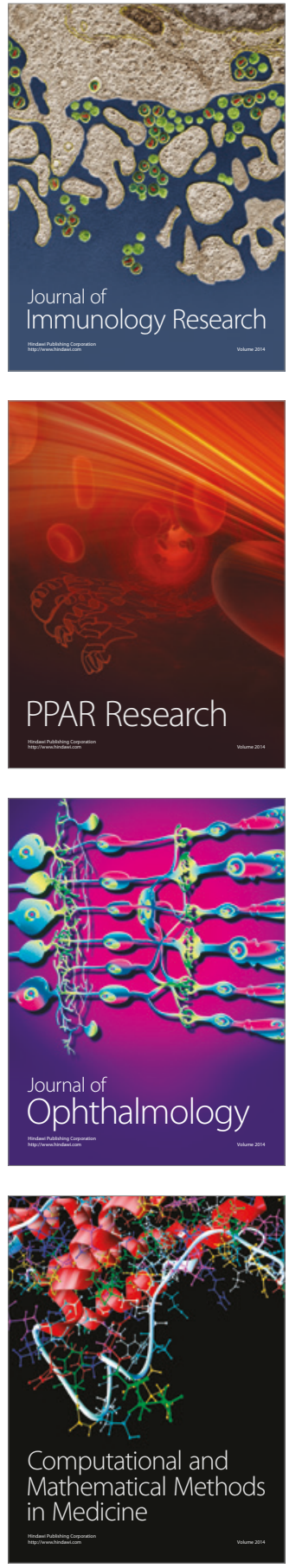

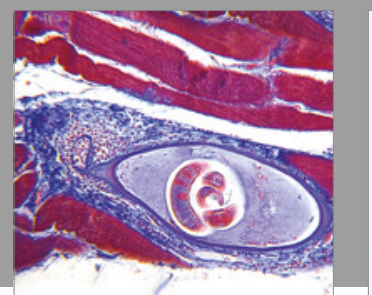

Gastroenterology Research and Practice
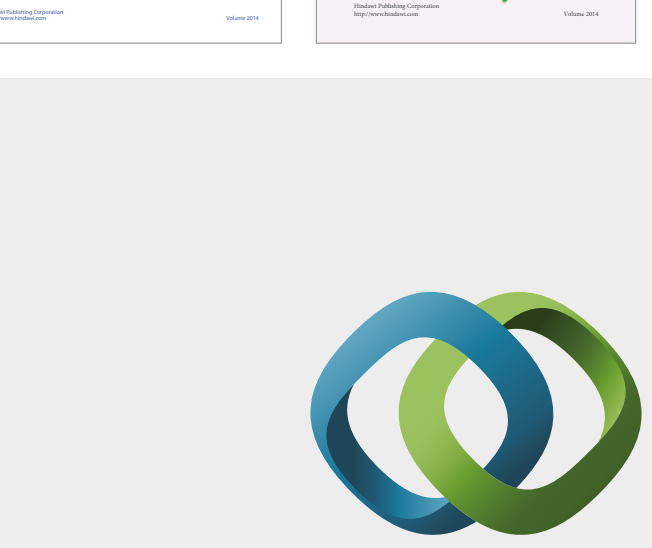

\section{Hindawi}

Submit your manuscripts at

https://www.hindawi.com
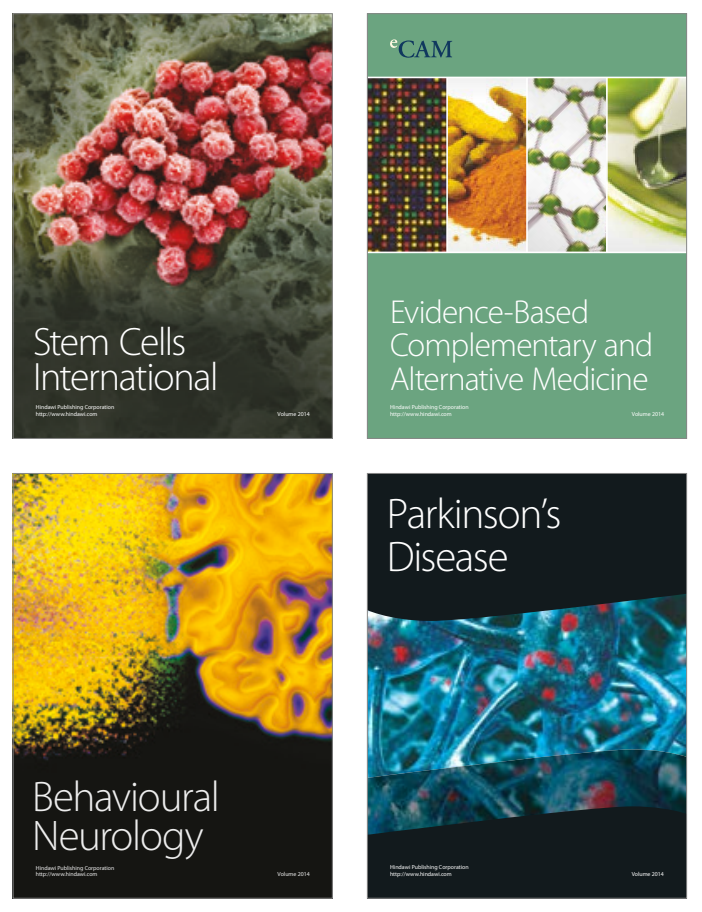
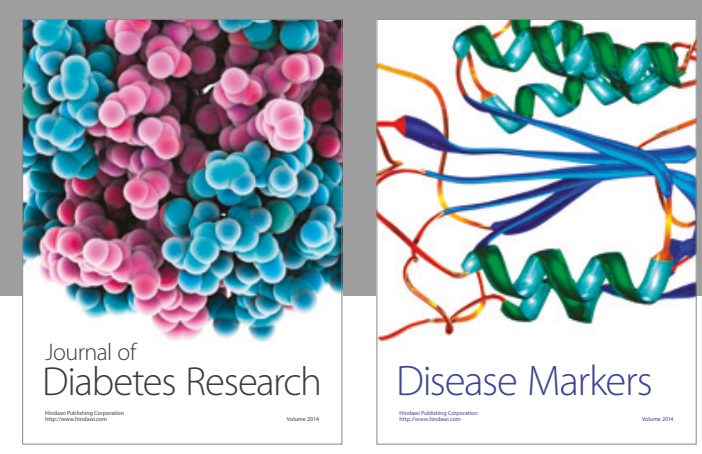

Disease Markers
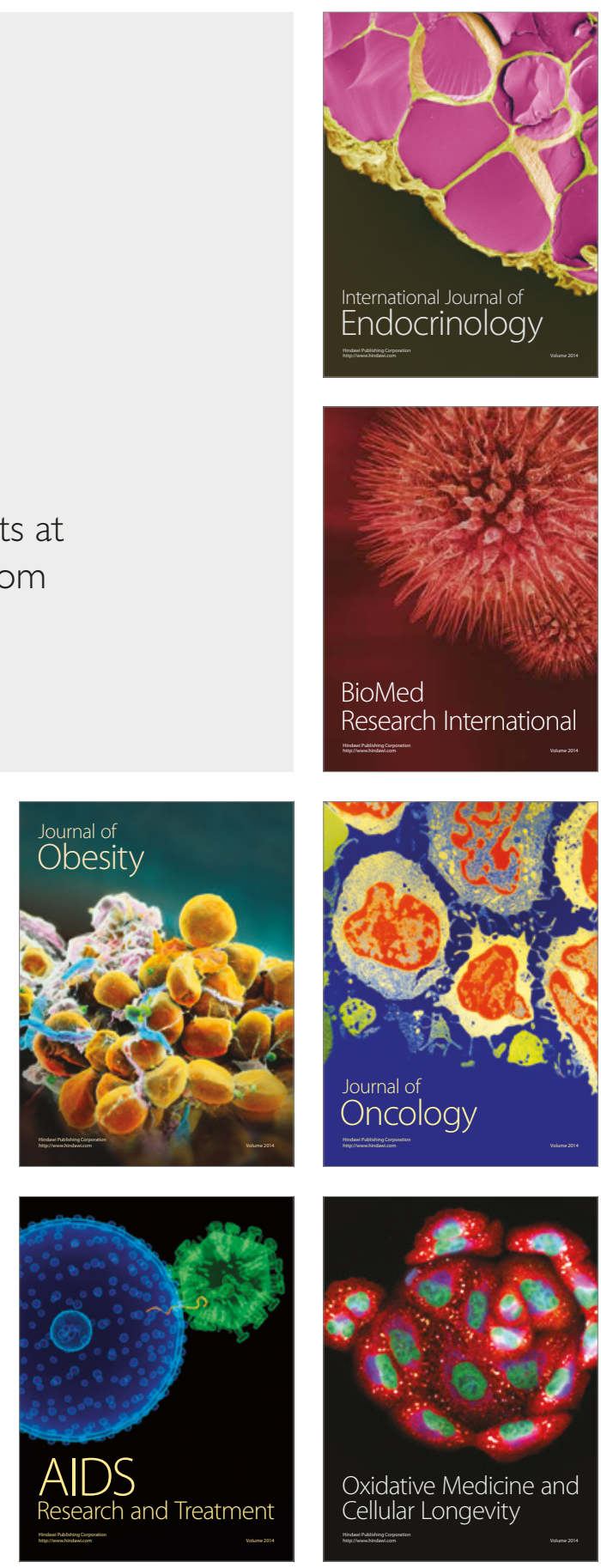